\title{
TIPOGRAFIA EM MOVIMENTO: UMA REVISÃO SISTEMÁTICA DE LITERATURA
}

Maíra Woloszyn

Universidade Federal de Santa Catarina

maira.projetar@gmail.com

Berenice Santos Gonçalves

Universidade Federal de Santa Catarina

beni.gon@gmail.com

Alice Theresinha Cybis Pereira

Universidade Federal de Santa Catarina

acybis@gmail.com

Milton Luiz Horn Vieira

Universidade Federal de Santa Catarina

mlvieira@mbox1.ufsc.br

Resumo: O desenvolvimento da tecnologia está tornando a transmissão de informação mais atrativa, eficiente e rápida. Com isso, as formas tradicionais de comunicação estão se adaptando e se reinventando para atender essa demanda. Um exemplo disso é a tipografia, muito utilizada para compor textos em publicações impressas. Combinada com o movimento, vem ganhando destaque em vários meios de comunicação digitais como cinema, televisão, vídeos, páginas da internet, livros digitais, entre outros. Diante deste contexto, o presente artigo busca compreender os principais fundamentos da tipografia em movimento. Para tanto, adotou-se como método uma revisão sistemática de literatura a respeito do tema, que permite sintetizar o conhecimento disponível sobre um determinado assunto e direcioná-lo para aplicação. A partir dos resultados alcançados nesse processo, são apresentados os principais apontamentos encontrados nos documentos analisados. Ainda, propõem-se a sistematização dos princípios de configuração da tipografia em movimento, desenvolvida com base nas discussões dos resultados.

Palavras-chave: tipografia, movimento, revisão sistemática.

Abstract: The development of technology is making transmission of information more attractive, fast and efficient. Thus, the traditional forms of communication are adapting and reinventing itself to meet this demand. An example is the typography, frequently used to compose texts in printed publications. When combined with movement, is outstanding in various digital media such as film, television, videos, websites, digital books, and others. Thereby, this paper seeks to understand the key fundamentals of 
typography in motion. For this, was performed a systematic review of literature on the subject, that provides synthesize of the available research on a particular topic and direct it to application. From the results, presented the main notes found in the analyzed documents. Also, propose to arrange the principles of typography in motion, based on the discussions of the results.

Key-words: typography, motion, systematic review.

\section{INTRODUÇÃO}

Para Samara (2011, p. 6) "a tipografia nos envolve em todos os momentos do dia - dos mundanos itens listados nas contas que pagamos e nas etiquetas de alimentos aos bombardeios das experiências alfabéticas em cartazes, na Internet e na TV". Ela é uma ferramenta fundamental na comunicação visual e vem se transformando em resposta às inovações tecnológicas e à evolução cultural. O avanço da tecnologia digital está melhorando o potencial para a comunicação e proporcionando representar conceitos de forma visual e expressiva. Um resultado disto é a combinação da tipografia com movimento que, diferente de sua forma estática, baseada em impressão, pode integrar diferentes conceitos como o de tecnologia, música, narrativa literária, entre outros (HOSTETLER, 2006).

A tipografia em movimento pode ser configurada simplesmente a partir da rolagem de um texto, como em créditos de filmes, ou textos que percorrerem diversos caminhos e mudam de lugar na tela (BROWNIE, 2007). Ainda, ela não precisa se mover literalmente, as palavras e letras podem "aparecer e desaparecer, piscar letra por leta, ou mudar de escala, cor, camada e assim por diante" (LUPTON; PHILLIPS, 2008, p. 226).

Segundo Lupton (2015, p. 165), este recurso visual "domina telas de todos os tipos e tamanhos, em todas as plataformas digitais, de celulares a computadores e telões gigantes". Smeijers (2015) afirma que a tela se tornou importante suporte de informação que nos permite apresentar o conteúdo, movimentando e modificando imagens e textos, através de diversos produtos multimídia. Esses produtos comunicam e transmitem o conteúdo a partir da integração de duas ou mais mídias - gráficos, animações, música, vídeo, fala e texto - e são apresentados através de um dispositivo digital - computador, tablet, smartphone (PAULA FILHO, 2009).

Publicações que requem o uso de um dispositivo digital, normalmente são apresentadas por meio de uma interface, entendida como "o espaço no qual se estrutura a interação entre corpo, ferramenta (objeto ou signo) e objetivo da ação. (...) A interface revela o conteúdo comunicativo das informações" (BONSIEPE, 1997, p. 12), e sua função é "permitir ao usuário uma visão panorâmica do conteúdo, navegar na massa de dados sem perder a orientação e, por fim, mover-se no espaço informacional de acordo com seus interesses" (BONSIEPE, 1997, p. 59). Nesse sentido, vários recursos gráficos, inclusive a tipografia, podem auxiliar os usuários a navegar pela interface, que, conforme explica Passos (2008, p. 33), oferece diversos fluxos de informação "permitindo novas conexões e consecutivamente, novas possibilidades" de comunicação, como é a tipografia combinada com movimento.

É possível perceber o uso da tipografia em movimento principalmente em créditos de filmes e clipes musicais. Ainda, é recorrente o uso desse recurso em outros 
produtos como livros digitais, telões urbanos e até mesmo de formas mais sutis com tipos que se movem ao abrir aplicativos de smartphones ou tablets (LUPTON, 2015). Com isso, não há duvidas que o movimento "dá vida a tipografia ao adicionar complexidade e dimensão a letras bidimensionais" (LUPTON, 2015, p. 165). Entretanto, esta é uma abordagem recente no design e que necessita se aprofundada.

\section{PROCEDIMENTOS METODOLÓGICOS}

Este estudo realizou uma revisão sistemática de literatura a fim de identificar os recentes conhecimento produzidos sobre tipografia em movimento, suas abordagens e princípios. Este método de pesquisa propõe a aplicação de estratégias científicas para a busca, seleção e avaliação de artigos, que serão sintetizados em um estudo específico. Ainda, tem por princípios gerais a exaustão na busca de artigos, a seleção justificada por critérios de inclusão e exclusão de documentos e, a quantificação dos resultados. Suas etapas são: (i) Identificação do tema e seleção da hipótese ou questão de pesquisa; (ii) Estabelecimento de critérios para inclusão ou exclusão de estudos; (iii) Categorização dos estudos; (iv) Avaliação dos estudos selecionados; (v) Interpretação dos resultados; (vi) Apresentação da síntese da revisão sistemática (MENDES, SILVEIRA, GALVÃO, 2008), conforme mostra o esquema a seguir.

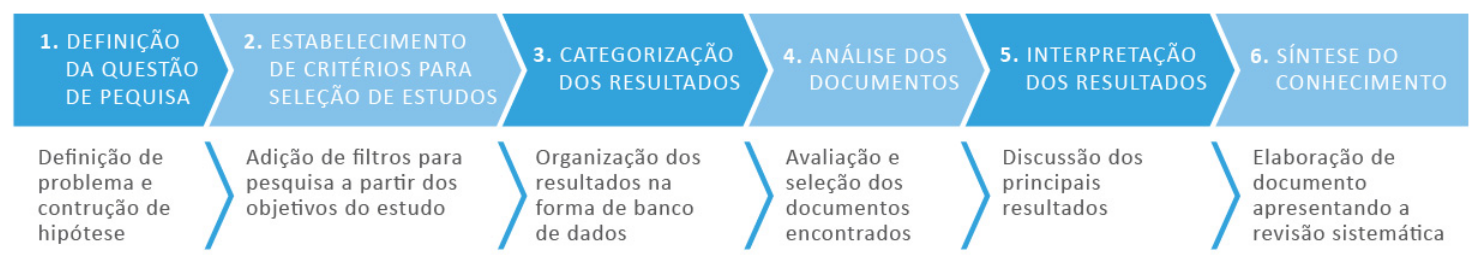

Figura 1 - Etapas da revisão sistemática.

Fonte: Elaborado pelos autores, com base em Mendes, Silveira e Galvão (2008).

Com base na questão da pesquisa - identificar abordagens e princípios da tipografia em movimento -, foi estabelecida a estratégia de busca a partir de dois eixos relevantes ao estudo: tipografia e movimento. A seguir, foram geradas alternativas de termos relacionados, todos em inglês, para a definição final da estratégia, conforme mostra a tabela abaixo.

\begin{tabular}{|l|l|}
\hline TIPOGRAFIA & MOVIMENTO \\
\hline Typography & Movement \\
\hline & Move \\
\hline & Motion \\
\hline & Animation \\
\hline & Kinetic \\
\hline
\end{tabular}

Tabela 1 - Eixos da pesquisa.

Fonte: Elaborado pelos autores.

Para ligar os termos sinônimos do eixo de movimento, foi utilizado o operador booleano "OR", já para ligar os dois eixos, "tipografia" e "movimento", o operador "AND". Desta forma, definiu-se a estratégia de pesquisa: "(typography AND (movement OR move OR motion OR animation OR kinetic))". A partir disso, 
escolheram-se as bases de dados onde à pesquisa foi submetida. Pela recorrência na área de Ciências Sociais Aplicadas, selecionou-se as bases Scopus, Web of Science e Science Direct. Posteriormente, aplicou-se a mesma estratégia de pesquisa na base de dados ACM Digital Library, reconhecida como um repositório de documentos em texto completo publicados pela Association for Computing Machinery (ACM) e entidades associadas, com artigos de pesquisa nas áreas de computação e tecnologia da informação (CAPES, 2016).

Sem aplicar filtros para a pesquisa, a busca gerou um resultado de 180 documentos na base Scopus, 220 na Web of Science, 1707 na Science Direct e 6 na ACM Digital Library, conforme exposto no gráfico a seguir.

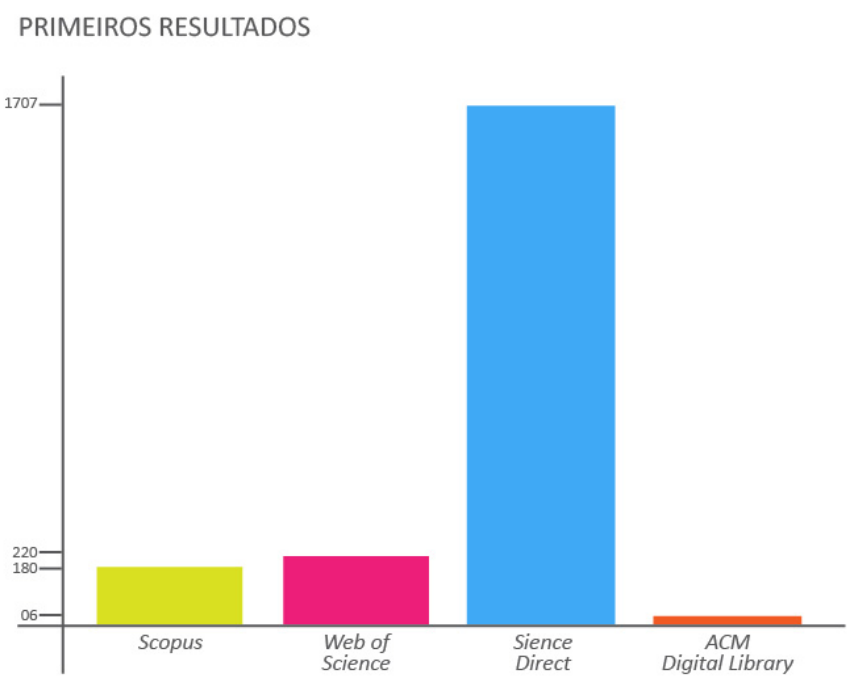

Figura 2 - Gráfico dos resultados quantitativos alcançados nas pesquisas.

Fonte: Elaborado pelos autores, com base na pesquisa realizada.

Após a pesquisa prévia, definiu-se que seriam utilizados apenas artigos publicados nos últimos dez anos, com isso, resultou em 54 artigos na base Scopus, 129 na Web of Science, 325 na Science Direct e 3 na ACM Digital Library, conforme mostra o gráfico a seguir.

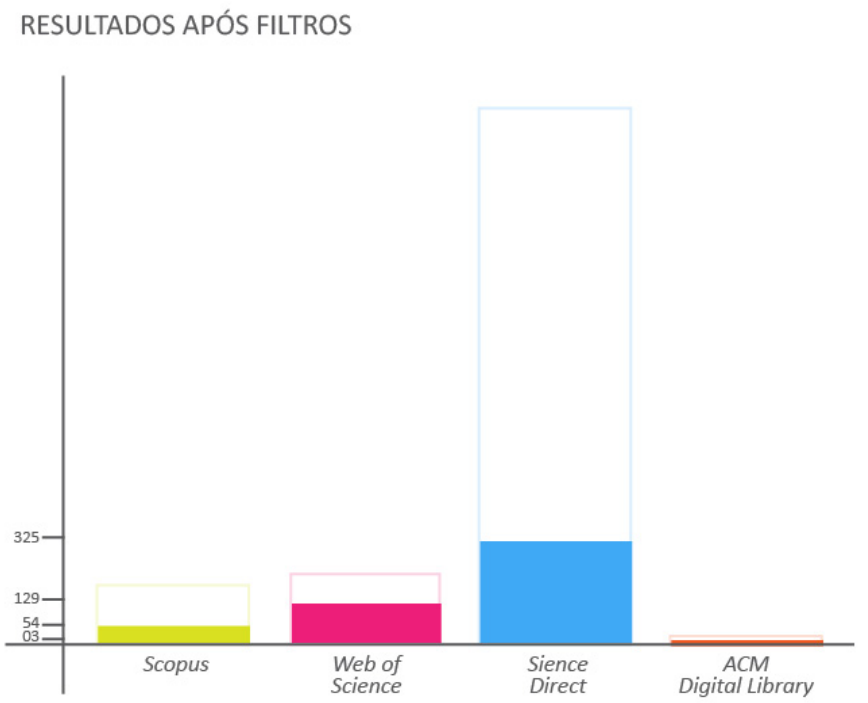

Figura 3 - Gráfico dos resultados quantitativos alcançados nas pesquisas após filtros.

Fonte: Elaborado pelos autores, com base na pesquisa realizada. 
Esses resultados foram exportados para o gerenciador bibliográfico Mendeley, que organiza os documentos em uma tabela com diversas informações como autor, título do artigo, ano e local de publicação. Ainda é possível visualizar o resumo e o link para acesso do documento completo na mesma plataforma, conforme mostra a figura a seguir. Esta ferramenta também permitiu identificar os documentos que estivessem duplicados, os quais foram excluídos, restando 312 artigos.

\begin{tabular}{|c|c|c|c|c|c|c|}
\hline$\star$ & - 自 & Authors & Title & Year & Published In & Details \\
\hline th & - & Abdel-Galli, Rani... & $\begin{array}{l}\text { Desert reclamation, a management } \\
\text { system for sustainable urban expa... }\end{array}$ & 2012 & $\begin{array}{l}\text { Progress in } \\
\text { Planning }\end{array}$ & \multirow{5}{*}{$\begin{array}{l}\text { Type: Journal Article } \\
\text { Desert reclamation, a management } \\
\text { system for sustainable urban expan... } \\
\text { Authors: R. Abdel-Galil } \\
\qquad \mathbb{N} \text { View research catalog entry for this paper }\end{array}$} \\
\hline 列 & $\bullet$ & Aboim Borges, M... & $\begin{array}{l}\text { User-sensing as Part of a } \\
\text { Wayfinding Design Process }\end{array}$ & 2015 & $\begin{array}{l}\text { Procedia } \\
\text { Manufacturing }\end{array}$ & \\
\hline 视 & $\bullet$ & Abson, D J; von ... & $\begin{array}{l}\text { Ecosystem services as a boundary } \\
\text { object for sustainability }\end{array}$ & 2014 & $\begin{array}{l}\text { Ecological } \\
\text { Economics }\end{array}$ & \\
\hline$\hbar$ & $\bullet$ & Adsanatham, Ch... & $\begin{array}{l}\text { Integrating Assessment and } \\
\text { Instruction: Using Student-Gener... }\end{array}$ & 2012 & $\begin{array}{l}\text { Computers and } \\
\text { Composition }\end{array}$ & \\
\hline tr & $\bullet$ & Aizpurua, Amaia... & $\begin{array}{l}\text { Prejudices, memories, expectations } \\
\text { and confidence influence experien... }\end{array}$ & 2015 & $\begin{array}{l}\text { Computers in } \\
\text { Human Beha... }\end{array}$ & \\
\hline t & $\bullet$ & Akbulut, Dilek & $\begin{array}{l}\text { The effects of different student } \\
\text { backgrounds in basic design educ... }\end{array}$ & 2010 & $\begin{array}{l}\text { Procedia - } \\
\text { Social and Be... }\end{array}$ & \multirow{10}{*}{ 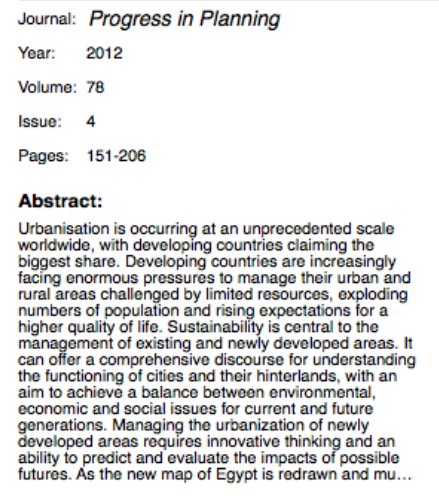 } \\
\hline th & $\bullet$ & Altin Gumussoy, ... & $\begin{array}{l}\text { Usability guideline for banking } \\
\text { software design }\end{array}$ & 2016 & $\begin{array}{l}\text { Computers in } \\
\text { Human Beha... }\end{array}$ & \\
\hline tr & $\bullet$ & Amato, Flora; D... & $\begin{array}{l}\text { An integrated framework for } \\
\text { securing semi-structured health re... }\end{array}$ & 2015 & $\begin{array}{l}\text { Knowledge- } \\
\text { Based Systems }\end{array}$ & \\
\hline 光 & $\bullet$ & Amaya-Castella... & $\begin{array}{l}\text { Development of an educational } \\
\text { intervention to promote healthy ea... }\end{array}$ & 2015 & $\begin{array}{l}\text { Evaluation and } \\
\text { Program Plan... }\end{array}$ & \\
\hline t & $\bullet$ & Arditi, A; Cho, J & $\begin{array}{l}\text { Letter case and text legibility in } \\
\text { normal and low vision }\end{array}$ & 2007 & $\begin{array}{l}\text { Vision } \\
\text { Research }\end{array}$ & \\
\hline is & $\bullet$ & Ariño, Joaquin; A... & $\begin{array}{l}\text { Chapter One - Systems Biology of } \\
\text { Monovalent Cation Homeostasis } \mathrm{i} . .\end{array}$ & 2014 & $\begin{array}{l}\text { Advances in } \\
\text { Microbial Syst... }\end{array}$ & \\
\hline$\hbar$ & $\bullet$ & Ayars, James e.; ... & 1. Introduction & 2007 & $\begin{array}{l}\text { Microirrigation } \\
\text { for Crop Prod... }\end{array}$ & \\
\hline 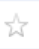 & $\bullet$ & Azmi, Aqil M; Als... & $\begin{array}{l}\text { A calligraphic based scheme to } \\
\text { justify Arabic text improving reada... }\end{array}$ & 2014 & $\begin{array}{l}\text { Computers in } \\
\text { Human Beha... }\end{array}$ & \\
\hline t & $\bullet$ & Bachfischer, G; R... & $\begin{array}{l}\text { Understanding influences of the } \\
\text { typographic quality of text }\end{array}$ & 2007 & $\begin{array}{l}\text { Journal of } \\
\text { Internet Com... }\end{array}$ & \\
\hline tis & $\bullet$ & Badea, Tudor Co... & $\begin{array}{l}\text { Morphologies of mouse retinal } \\
\text { ganglion cells expressing transcrip... }\end{array}$ & 2011 & $\begin{array}{l}\text { Vision } \\
\text { Research }\end{array}$ & \\
\hline tr & $\bullet$ & Balsa-Canto, Ev... & $\begin{array}{l}\text { Optimal design of dynamic } \\
\text { experiments for improved estimati... }\end{array}$ & 2007 & $\begin{array}{l}\text { Journal of Food } \\
\text { Engineering }\end{array}$ & \multirow{5}{*}{$\begin{array}{l}\text { Tags: } \\
\text { Author Keywords: } \\
\text { Developing countries; Indicators; Scenarios; } \\
\text { Sustainable urban management; Targets } \\
\text { Month: } \\
\text { novembro }\end{array}$} \\
\hline t & - & Barratt, Monica ... & $\begin{array}{l}\text { 'What if you live on top of a bakery } \\
\text { and you like cakes?'-Drug use a... }\end{array}$ & & $\begin{array}{l}\text { International } \\
\text { Journal of Dr... }\end{array}$ & \\
\hline tr & $\bullet$ & Barth, $\mathrm{S}$ & $\begin{array}{l}\text { Digital designs: A look at the role of } \\
\text { real typographic design in effectiv... }\end{array}$ & 2008 & EContent & \\
\hline$\hbar$ & $\bullet$ & Barton, Gabriel & $\begin{array}{l}\text { Monolayers polarizable } \\
\text { perpendicularly: The Maxwellian r... }\end{array}$ & 2015 & $\begin{array}{l}\text { Annals of } \\
\text { Physics }\end{array}$ & \\
\hline A & - & Bav-Chena. S & Translation. tvooaraohv. and the & 2007 & Theatre Journal & \\
\hline
\end{tabular}

Figura 4 - Gerenciador bibliográfico Mendeley.

Fonte: Aplicativo para desktop Mendeley, 2016.

Destes, ainda foram excluídos os artigos que não estivessem nos idiomas inglês, português ou espanhol.

Após a leitura dos títulos e resumos, foram selecionados doze artigos para serem analisados na íntegra. Conforme mostra o quadro a seguir, destes, três documentos, marcados em vermelho, não apresentavam relação com a tipografia em movimento. Quatro, marcados em amarelo, se aproximavam do assunto, porém não traziam esclarecimentos sobre o tema. E, apenas cinco, marcados em verde, apresentavam contribuições que iam ao encontro da temática proposta, e serão discutidos a seguir. 


\begin{tabular}{|c|c|c|}
\hline Autor & Título & Ano \\
\hline Adérito Fernandes Marcos & $\begin{array}{l}\text { Editorial of the special issue "Technology and digital } \\
\text { art" }\end{array}$ & 2007 \\
\hline $\begin{array}{l}\text { Dmitry A. Tarasova, } \\
\text { Alexander P. Sergeeva, } \\
\text { Victor V. Filimonova }\end{array}$ & Legibility of textbooks: a literature review & 2015 \\
\hline João Aranda Brandão & $\begin{array}{l}\text { Motion Graphics Ergonomics: Animated Semantic } \\
\text { System, for Typographical Communication Efficiency }\end{array}$ & 2015 \\
\hline $\begin{array}{l}\text { Jonathan Alexander, Julia R. } \\
\text { Lupton }\end{array}$ & Letters from the editor & 2014 \\
\hline $\begin{array}{l}\text { Joonhawan Lee, Soojin Jun, } \\
\text { Jodi Forlizzi, Scott E. } \\
\text { Hudson }\end{array}$ & $\begin{array}{l}\text { Using Kinetic Typography to Convey Emotion in Text- } \\
\text { Based Interpersonal Communication }\end{array}$ & 2006 \\
\hline $\begin{array}{l}\text { Luiz Fernando } \\
\text { Las-Casas }\end{array}$ & Cinedesign: typography in motion pictures & 2007 \\
\hline Mohamed Zaki Ramadan & $\begin{array}{l}\text { Evaluating college students' performance of Arabic } \\
\text { typeface style, font size, page layout and } \\
\text { foreground/background color combinations dføok } \\
\text { materials }\end{array}$ & 2011 \\
\hline $\begin{array}{l}\text { Oliviero Stock, } \\
\text { Carlo Strapparava, } \\
\text { Alessandro Valitutti }\end{array}$ & Ironic expressions and moving words & 2008 \\
\hline Ozden Pektas Turgut & Kinetic typography in movie title sequences & 2012 \\
\hline $\begin{array}{l}\text { Sabrina Malik, } \\
\text { Jonathan Aitken, } \\
\text { Judith Kelly Waalen }\end{array}$ & Communicating emotion with animated text & 2009 \\
\hline Sung-Hee Jin & $\begin{array}{l}\text { Visual design guidelines for improving learning from } \\
\text { dynamic and interactive digital text }\end{array}$ & 2013 \\
\hline Theo van Leeuwen & Towards a semiotic of typography & 2006 \\
\hline
\end{tabular}

Quadro 1 - Artigos analisados na íntegra onde as linhas marcadas em verde apontam os estudos que tratam da temática proposta e que foram selecionados para serem discutidos.

Fonte: Elaborado pelos autores.

\section{RESULTADOS E DISCUSSÕES}

Conforme apresentado anteriormente, a tipografia em movimento também é resultado dos avanços da tecnologia e vem sendo explorada em diversos produtos multimídia, mas ainda é um tema de abordagem recente para o design. De acordo com Las-Casas (2007), a introdução do computador como ferramenta de trabalho na cultura promoveu novas formas de comunicação e entretenimento e implicou em uma transformação da produção do design gráfico. Com isso, a natureza audiovisual se tornou cada vez mais presente nos meios de comunicação, mas ainda com uma estrutura informacional baseada na fala e na escrita.

Como exposto por Turgut (2012), com as novas tecnologias, a tipografia foi removida dos limites da publicação impressa e inserida para uso efetivo em projetos de diferentes meios de comunicação, como a televisão, vídeo, cinema, web, entre outros. Essas tecnologias permitiram mudar o entendimento já conhecido da 
tipografia, dando lugar a textos e tipos animados, que são mais expressivos do que os utilizados de forma impressa, compreendidos como tipografia em movimento ou tipografia cinética. Lee, Jun, Forlizzi e Hudson (2006) definem a tipografia em movimento como o texto que muda de cor, tamanho ou posição ao longo do tempo. Leeuwen (2006) ainda sugere que a tipografia está enfrentando novos desafios com as mídias baseadas em tela, uma vez que as publicações estão se tornando cada vez mais visuais.

Diante deste contexto, Turgut (2012) ressalta que a tipografia não é apenas um meio de transmitir uma informação ou mensagem de forma compreensível, mas também é um elemento gráfico que traduz estilo, personalidade e linguagem visual. Por isso, cada fonte tem suas características específicas que pode refletir o significado de uma mensagem ou de uma imagem. Assim, a escolha da fonte para um produto pode reforçar o conteúdo que está sendo transmitido ou até mesmo causar confusão para o usuário.

Ainda, Leeuwen (2006) explica que a tipografia não trata somente sobre a forma e composição das letras. Ela se apresenta integrada com outros meios tais como cor, textura e tridimensionalidade, que também devem ser observados junto com os tipos, uma vez que podem destacar ou não uma informação em um ambiente visual e expressar significados diferentes, principalmente se tratando da cor. Também é importante não isolar a tipografia dos demais elementos gráficos apresentados juntos a ela em um mesmo produto, como ilustrações, fotografia, background, etc, uma vez que essas informações distintas se interligam de muitas maneiras diferentes.

Assim, a tipografia pode promover interações e expressar atitudes. Uma palavra pode ter seu sentido modificado apenas por meio da tipografia ou por sinais tipográficos (LEEUWEN, 2006). Essas atitudes também são proporcionadas a partir da combinação da tipografia com movimento pois, como acredita Brandão (2015), o movimento contribui para moldar o texto e conferir a ele características importantes e sugestivas. Para o autor, projetos desenvolvidos com uma linguagem visual dinâmica permite uma maior eficiência na comunicação. O autor ainda cita Rudolf Arnhein ao afirmar que "o movimento é o apelo mais forte à atenção" (1997, apud BRANDÃO, 2015, p. 6377, tradução nossa). Nesse sentido, Lee, Jun, Forlizzi e Hudson (2006), ainda afirmam que a adição do movimento à tipografia pode expressar emoções na comunicação baseada em texto. Além disso, o movimento pode conferir hierarquia às informações de um produto e, também, narrar histórias e expressar sentimentos (BRANDÃO, 2015).

Não há dúvidas que o movimento é um forte elemento de interação para a tipografia, e isso fica ainda mais evidente quando Brandão (2015) apresenta o resultado de testes desenvolvidos com tipografia em movimento. Segundo ele, após os testes, foi concluído que os leitores prestam mais atenção no texto em movimento do que em qualquer outro elemento presente na tela. Ao utilizarmos a tipografia em movimento, podemos mudar a percepção do usuário sobre a mensagem e também modificar o significado delas, uma vez que ela pode substituir pontuações, dar ênfase, e indicar pausas e divisões através do ritmo (BRANDÃO, 2015). Como abordado por Las-Casa (2007), o ritmo depende da articulação e integração de todos os componentes de um produto visual para garantir fluidez da sequencia de imagens, sons e textos que se apresentam ao mesmo tempo. 
Para Las-Casas (2007), a principal diferença entre o design para materiais impressos e para produtos digitais é a adição de um elemento temporal incorporado na sua narrativa. Pois, em peças impressas, o elemento "tempo" é determinado pelo espectador, é ele quem determina quando e por quanto tempo irá folhear ou ler uma revista, por exemplo. Desta forma, ao adicionar "tempo" como um elemento de design à tipografia estática, tem-se o texto dinâmico nas mídias digitais (LEE; JUN; FORLIZZI; HUDSON, 2006).

Nessa perspectiva, pode-se afirmar que em um projeto de tipografia em movimento é possível observar tanto as questões tipográficas, quanto a relação com os elementos integrados à tipografia. Turgut (2012) ainda acrescenta que um ponto a ser observado neste tipo de projeto é o planejamento dos espaços e a organização dos elementos apresentado. Ainda, Brandão (2015) acredita que a hierarquia visual de um produto orienta os usuários e os ajuda a compreender quais as informações mais importantes da mensagem apresentada. Ele afirma que uma distinção clara entre as informações primárias, secundárias e terciárias, é o que define uma forte hierarquia visual. Conforme explica Turgut (2012), uma organização eficaz da tipografia em movimento reforça a ligação emocional do público com o produto.

A partir deste levantamento, foi possível identificar os principais fatores, abordados pelos autores, que devem ser observados na construção de um projeto de tipografia em movimento. Esses resultados foram sistematizados conforme o quadro a seguir.

\begin{tabular}{|l|l|l|}
\hline Autor & Ano & Contribuição \\
\hline BRANDÃO & 2015 & $\begin{array}{l}\text { O movimento é o apelo mais forte à atenção e contribui para direcionar ao } \\
\text { texto características e significados importantes. Por conta do ritmo, o } \\
\text { movimento pode servir como ferramenta semântica, trazendo ênfases às } \\
\text { mensagens e mudando a percepção do usuário sobre elas, e também organizar } \\
\text { a hierarquia visual do produto que utiliza a tipografia em movimento. }\end{array}$ \\
\hline LAS-CASAS & 2007 & $\begin{array}{l}\text { A principal diferença de produtos impressos para os digitais é a adição de um } \\
\text { elemento temporal em sua narrativa, o que, em produtos digitais, diz respeito } \\
\text { ao ritmo em que as informações são apresentadas. } \\
\text { Oritmo diz respeito à integração de todos os elementos presentes de modo a } \\
\text { garantir fluidez para o produto. }\end{array}$ \\
\hline LEE; JUN; & 2006 & $\begin{array}{l}\text { A tipografia em movimento é definida como o texto que muda de cor, } \\
\text { tamanho ou posição ao longo do tempo. Considerando o tempo como um } \\
\text { elemento de design junto à tipografia estática, tem-se os textos dinâmicos das } \\
\text { mídias digitais. } \\
\text { Ao adicionar movimento à tipografia pode-se melhorar as qualidades } \\
\text { emocionais da comunicação baseada em texto. }\end{array}$ \\
\hline HUDSON & 2006 & $\begin{array}{l}\text { A tipografia não diz respeito apenas à forma das letras, ela trata também de } \\
\text { outros meios integrados à ela, como cor, textura e tridimensionalidade e } \\
\text { movimento. } \\
\text { É importante não isolar a tipografia das outras formas de comunicação que se } \\
\text { apresentam junto à ela, como plano de fundo, imagens e sons. }\end{array}$ \\
\hline TURGWT & 2012 & $\begin{array}{l}\text { A tipografia não se refere apenas à transmissão de mensagens de forma } \\
\text { compreensível, mas um elemento que apresenta estilo, personalidade e } \\
\text { linguagem visual. Cada fonte tem características próprias e específicas. } \\
\text { Deve-se planejar desde a seleção de fontes e o tamanho das letras, até a } \\
\text { organização visual do produto contemplando os espaços e localização do } \\
\text { percurso dos tipos. }\end{array}$ \\
\hline
\end{tabular}

Quadro 2 - Contribuições dos autores sobre o tema.

Fonte: Elaborado pelos autores, com base nos resultados da revisão sistemática. 
A partir desta sistematização, foi possível identificar os princípios de configuração da tipografia em movimento, abordado pelos autores, e a partir disso, propor a organização destes princípios em eixos, conforme apresentado na figura 5.0 primeiro, "Eixo tipográfico", está relacionado com aspectos formais dos tipos, como estilo tipográfico, tamanho, peso (light, médium, bold), espaçamento entre letras e linhas, entre outros. Também, é onde encontramos os elementos que estão integrados aos tipos, como a cor utilizadas, se existe alguma textura ou tridimensionalidade aplicada à letra.
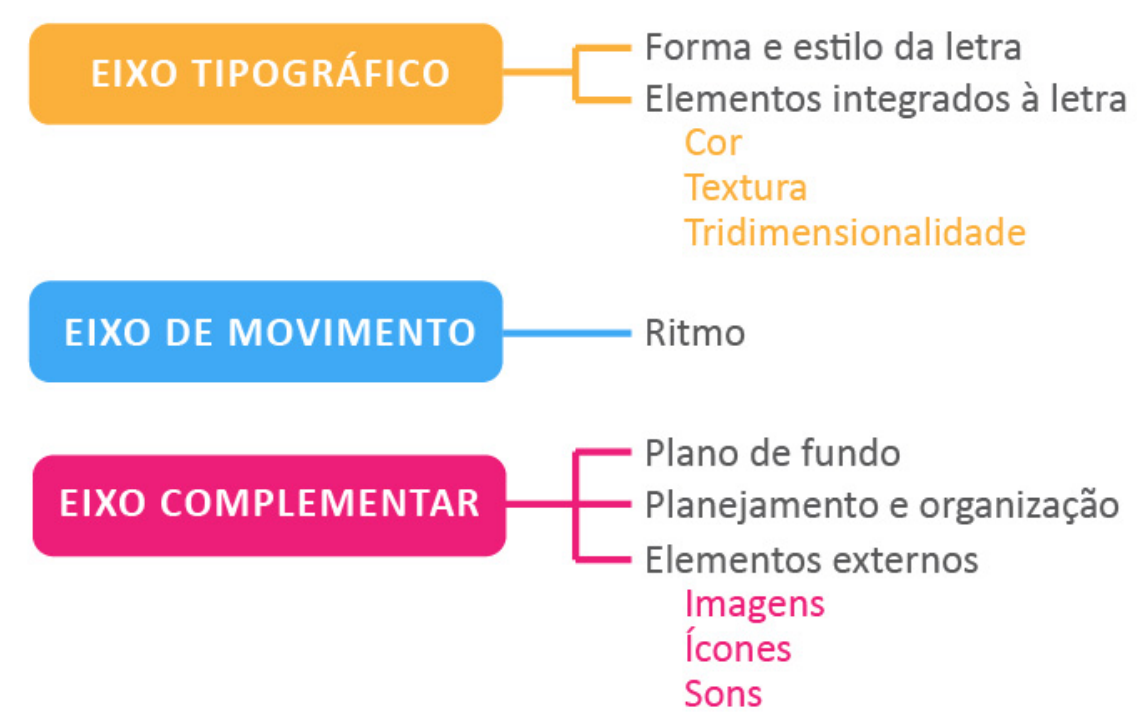

Figura 5 - Eixos de princípios de configuração da tipografia em movimento.

Fonte: Elaborado pelos autores, com base nos resultados da revisão sistemática.

O segundo eixo, trata dos aspectos relacionados com o movimento dos tipos, mais precisamente o ritmo. Ele diz respeito à fluidez da associação dos componentes do produto, e também determina o tempo e a velocidade que o usuário irá interagir com o produto.

Já no terceiro eixo, estão os elementos complementares à tipografia em movimento presente no produto. Trata-se do plano de fundo, se possui imagens, texturas ou cores. Contempla também o planejamento do espaço e como estão organizados os elementos presentes e hierarquizadas as informações. E por fim, observa os elementos externos à tipografia, como ilustrações, fotografias, ícones, sons e demais artefatos presentes.

\section{CONSIDERAÇÕES FINAIS}

$O$ avanço das tecnologias tem proporcionado o surgimento de novas formas de comunicação. Um exemplo disso, é a tipografia em movimento, que permite comunicar através do movimento dos tipos. Este estudo foi desenvolvido com o objetivo de identificar estudos recentes sobre o tema, o que foi possível através da análise dos resultados da revisão sistemática.

Apesar de inicialmente as buscas terem gerado um número expressivo de retornos, percebeu-se que muitos dos resultados não tinham direta relação com o tema ou apenas citavam o tema sem trazer esclarecimentos sobre o assunto. Da 
mesma forma, na segunda busca em uma nova base de dados, a ACM Digital Library, os poucos resultados obtidos não acrescentavam contribuições ao escopo do estudo. Nesse sentido, fica evidente que apesar de ser um recurso visual muito explorado pelos desenvolvedores de produtos digitais, o estudo acadêmico deste tema ainda é escasso. Contudo, o estudo permitiu identificar e sistematizar os princípios da tipografia em movimento, conforme pretendido no início desta pesquisa.

Após a análise dos resultados obtidos, percebe-se uma necessidade de ampliação de pesquisas, principalmente no Brasil, deste assunto, uma vez que todos os artigos que possuíam relação com o tema estavam publicados em inglês.

Como continuidade deste trabalho, sugere-se a evolução dos eixos desenvolvidos, juntamente de novos estudos e levantamentos, com o objetivo de construir parâmetros avaliativos da tipografia em movimento.

\section{REFERÊNCIAS}

BONSIEPE, Gui. Design: do material ao digital. Florianópolis: FIESC/IEL, 1997.

BRANDÃO, João Aranda. Motion Graphics Ergonomics: Animated Semantic System, for Typographical Communication Efficiency. Procedia manufacturing 3, 2015.

Disponível em <http://ac.els-cdn.com/S2351978915009658/1-s2.0-S2351978915009658-

main.pdf?_tid=b3bcfdda-149c-11e6-a560-

00000aacb35e\&acdnat=1462657574_da682fb8b1ce5d49be082fd4ce29c755> Acesso em 04 mai. 2016.

BROWNIE, Barbara. Fluid characters in temporal typhography. Fusion issue 1, 2012. Disponível em < http://www.fusion- journal.com/issue/001-fusion/fluid-characters-intemporal-typography/> Acesso em 30 mar. 2016.CAPES. Disponível em $<$ http://buscador-periodicos-capes-gov-

br.ez46.periodicos.capes.gov.br/V/NH64GXQIRCVTMY22KL3EA5NK6MB1V7FDH9B8YBKQ2ASI5 FLKXN-00841?func=find-db-info\&doc_num=000002598> Acesso em 10 ago 2016.

HILLNER, Matthias. Virtual Typography: time perception in relation to digital communication. Leonardo Electronic Almanac, 2006. Vol 14. No 05-06. Disponível em:<http://leoalmanac.org/journal/vol_14/lea_v14_n0506/mhillner_lea140506.html\# zoom $=100,0,0>$ Acesso em 30 mar. 2016

HOSTETLER, S.C. Integrating typography and motion in visual communication. Cedar Falls, IA, EUA: University of Northern lowa, 2006. Disponível em:

<http://www.units.muohio.edu/codeconference/paprs/papers/soo\%20hostetler20 06\%20idmaa\%20full\%20paper.pdf> Acesso em 30 mar. 2016.

LAS-CASA, Luiz Fernando. Cinedesign: typhography and motion pictures. InfoDesign, 2007. P. 12-19.

LEE, Joonhwan; JUN, Soojin; FORLIZZI, Jodi; HUDSON, Scott E. Using kinetic typography to convey emotion in text-based interpersonal communication. Proceedings of the 6th conference on Designing Interactive systems, 2006. Disponível em $<$ http://delivery.acm.org.ez46.periodicos.capes.gov.br/10.1145/1150000/1142414/p41lee. pdf?ip=200.130.19.174\&id=1142414\&acc=ACTIVE\%20SERVICE\&key=344E943C9DC262BB\% 2E6BDBE0C8DFC96E73\%2E4D4702B0C3E38B35\%2E4D4702B0C3E38B35\&CFID $=653985976 \& C$ FTOKEN $=74128314 \&$ _acm_ $=1470834013 \_50 b 65$ eb0b4851916fddecf246dd25467\#URLTOKE N\%23> Acesso em 10 ago 2016. 
LEEUWEN, Theo van. Towards a semiotics of typography. Information design journal, 2006. Disponível em

<http://www.ixdcth.se/courses/2012/tda492/sites/default/files/files/Reading_Towards_a_Se miotics_of_typography.pdf> Acesso em 04 mai. 2016.

LUPTON, Ellen. Tipos na tela. São Paulo: Gustavo Gili, 2015.

LUPTON, E.; PHILLIPS, J. C. Novos fundamentos do design. São Paulo: Cosac Naify, 2008.

MENDES, Karina Dal Sasso; SILVEIRA, Renata Cristina de Campos Pereira; GALVAO, Cristina Maria. Revisão integrativa: método de pesquisa para a incorporação de evidências na saúde e na enfermagem. Texto contexto - enferm., Florianópolis, v. 17, n. 4, 2008. p. 758-764.

PASSOS, Ravi Figueiredo. O Design da Informação em Interfaces de Hipermídias. 2008. 98 f. Dissertação de Mestrado - Universidade Anhembi Morumbi, São Paulo-SP, 2008. Disponível em: <http://www. anhembi.br/ppgdesign/pdfs/ravi.pdf>. Acesso em: 04 maio, 2016.

PAULA FILHO, Wilson de Pádua. Multimídia: conceitos e aplicações. Rio de Janeiro: LTC, 2009.

SAMARA, Timothy. Guia de tipografia. Porto Alegre: Bookman, 2011.

SMEIJERS, Fred. Contrapunção. Brasília: Estereográfica, 2015.

TURGUT, Ozden Pektas. Kinetic typography in movie title sequences. Artsedu, 2012. Disponível em http://www.sciencedirect.com/science/article/pii/S1877042812033472> Acesso em 04 mai. 2016. 